\title{
Bronchial thermoplasty: a new therapeutic option for the treatment of severe, uncontrolled asthma in adults
}

\author{
Marie-Christine Dombret ${ }^{1,8}$, Khuder Alagha2,8, Louis Philippe Boulet ${ }^{3}$, \\ Pierre Yves Brillet ${ }^{4}$, Guy Joos ${ }^{5}$, Michel Laviolette ${ }^{3}$, Renaud Louis ${ }^{6}$, \\ Thierry Rochat ${ }^{7}$, Paola Soccal ${ }^{7}$, Michel Aubier $^{1}$ and Pascal Chanez ${ }^{2}$ \\ Number 1 in the Series "Asthma" \\ Edited by Pascal Chanez and Marc Humbert
}

\begin{abstract}
Affiliations: 'Dept of Respiratory Medicine, Bichat APHP, U700 INSERM University Hospitals Dept, Diderot University, Paris, France. ${ }^{2}$ Dept of Respiratory Medicine, APHM, INSERM U1067 CNRS UMR 7333, Aix Marseille University, Marseille, France. ${ }^{3}$ Research Centre at the Institute of Cardiology and Respiratory Medicine, Laval University, Quebec, QC, Canada. ${ }^{4}$ Dept of Radiology, Avicenne Public Hospital, Bobigny, France. ${ }^{5}$ Dept of Respiratory Medicine, Ghent University Hospital, Ghent University, Ghent, Belgium. ${ }^{6}$ Dept of Respiratory Medicine, Liege University Hospital, GIGAI3 Research Group, University of Liège, Liège, Belgium. ${ }^{7}$ Dept of Respiratory Medicine, HUG Geneva, Switzerland. ${ }^{8}$ Both authors contributed equally.
\end{abstract}

Correspondence: Pascal Chanez, Dept of Respiratory Medicine, Marseille Public Hospitals, INSERM U1067 CNRS UMR 7333, Aix Marseille University, Hôpital Nord, 13015 Marseille, France.

E-mail: pascal.chanezQuniv-amu.fr

ABSTRACT Bronchial thermoplasty is a young yet promising treatment for severe asthma whose benefit for long-term asthma control outweighs the short-term risk of deterioration and hospitalisation in the days following the treatment. It is an innovative treatment whose clinical efficacy and safety are beginning to be better understood. Since this is a device-based therapy, the overall evaluation of risk-benefit is unlike that of pharmaceutical products; safety aspects, regulatory requirements, study design and effect size assessment may be unfamiliar. The mechanisms of action and optimal patient selection need to be addressed in further rigorous clinical and scientific studies. Bronchial thermoplasty fits in perfectly with the movement to expand personalised medicine in the field of chronic airway disorders. This is a device-based complimentary asthma treatment that must be supported and developed in order to meet the unmet needs of modern severe asthma management. The mechanisms of action and the type of patients that benefit from bronchial thermoplasty are the most important challenges for bronchial thermoplasty in the future.

@ERSpublications

Bronchial thermoplasty is a potential treatment for severe uncontrolled asthma in adults http://ow.ly/AgYVj

For editorial comments see page 405.

This article has supplementary material available from err.ersjournals.com

Received: June 112014 | Accepted after revision: July 032014

Conflict of interest: Disclosures can be found alongside the online version of this article at err.ersjournals.com

Provenance: Submitted article, peer reviewed.

Copyright OERS 2014. ERR articles are open access and distributed under the terms of the Creative Commons Attribution Non-Commercial Licence 4.0. 


\section{Introduction}

Severe asthma continues to represent a challenge for physicians. New treatment strategies and approaches are urgently needed for these patients [1]. Appropriate patient selection is paramount when considering any new treatment modality, be it pharmacological or device based. Bronchial thermoplasty is a nonpharmacological, device-based therapy that delivers controlled thermal energy to the airway wall as part of a series of three bronchoscopic procedures. It was approved by the US Food and Drug Administration for the treatment of severe persistent asthma in patients aged $\geqslant 18$ years whose asthma is not well controlled with inhaled corticosteroids (ICS) and long-acting $\beta_{2}$-agonists (LABAs) in 2010, and was CE marked and available in Europe since 2011. The clinical development programme consisted of five clinical trials; four were in subjects with asthma and three were randomised. The largest sham-controlled device-based clinical trial in severe asthma, the Asthma Intervention Research (AIR)2 Trial, has recently published 5-year efficacy and safety data [2]. Despite the large body of clinical work, many unresolved questions remain [3]. This review will summarise the information on mechanism of action, procedure, efficacy, safety and patient selection, to better understand the path forward for this promising technique.

\section{How do we perform bronchial thermoplasty?}

Bronchial thermoplasty is an interventional bronchoscopic procedure for the treatment of severe, uncontrolled asthma patients. Although simple in principle, correct procedure execution requires accurate and meticulous identification of airway anatomy via bronchoscopy. It should only be performed by an experienced bronchoscopist in a healthcare setting with appropriate clinical monitoring and the ability to address any potential post-intervention complications. The appropriate assessment and monitoring of the patient before, during and after the procedure has been described by MAYSE et al. [4].

Bronchial thermoplasty must only be offered to patients with documented asthma. At present, warnings, precautions and exclusions include chronic obstructive pulmonary disease, bronchiectasis, recurrent respiratory infections or any other uncontrolled significant respiratory disease. The treating facility (asthma specialist and interventional bronchoscopist) should be familiar with the patient and his/her clinical course in order to ensure appropriate patient selection and optimal asthma control prior to bronchial thermoplasty and during the entire treatment period. Treatment with bronchial thermoplasty can, in fact, lead to temporary worsening of asthma symptoms, the intensity of which may be proportional to the severity of the patient's asthma [5-7]. Peri-procedure systemic corticosteroid therapy, admission to hospital for intensive respiratory monitoring and/or bronchoscopy may be necessary. At the end of the treatment period ( 6 weeks after the final bronchoscopy), the clinical benefit should ideally be reassessed. Pharmacological treatments should be adjusted to their lowest possible dosages, starting with a reduction of oral corticosteroids (OCS) and followed by a reduction in ICS and/or LABA if the asthma remains well controlled [8]. There are no data on the effects of bronchial thermoplasty on airway inflammation to empirically guide pharmacological treatment, nor are there data on the effects of medication withdrawal following bronchial thermoplasty in a forced medication withdrawal study design other than a small safety study in very severe asthma patients [5].

\section{The procedure in practice}

Performing bronchial thermoplasty requires bronchoscopic rigour, dexterity and a thorough knowledge of the airway anatomy. Three treatment sessions at approximately 3 -week intervals are recommended; full recovery of the patient between treatments is necessary in order to proceed. The sequence of treatments involves the right lower lobe (first session) then left lower lobe (second session), followed by both upper lobes (third session). The right middle lobe is not treated, as the clinical programme excluded this area based on the theoretical possibility of obstruction and right middle lobe syndrome. Each bronchial thermoplasty session takes $\sim 30-45 \mathrm{~min}$. Each bronchus is treated along its entire visible length, with each activation targeting a 5-mm section of bronchus between 3 and $10 \mathrm{~mm}$ in diameter, beginning at the periphery and moving proximally. Areas should not be retreated. A full treatment consists of $\sim 30-70$ activations per lobe (depending on the specific anatomy); on average, 44 for the right lower lobe, 47 for the left lower lobe and 60 for the upper lobes are performed [9]. The effectiveness of the treatment may depend on how thoroughly the procedure is performed; if a segment is left untreated it may theoretically continue to constrict when stimulated, potentially negating the benefits of the treatment. This is why meticulous technique and airway mapping is so important. Activation of the bronchial thermoplasty catheter (fig. 1) does not produce any macroscopic effect on the bronchial mucosa but may result in transient blanching represented by a whitening of the mucosa. This makes it difficult to identify the treated bronchi and requires close attention on the part of the bronchoscopist in order to know which segments have been treated. As a result, in contrast to other procedures (such as laser treatment of an obstructive lesion, ablation of a mucous blockage or foreign body, etc.), there is no visible indication of treatment. Close clinical monitoring is required immediately following the procedure, as asthma symptoms are more common in the 
hours immediately following treatment. Bronchial thermoplasty requires great patience if there are abundant secretions or uncontrollable cough, when the airways move on inspiration (especially in the lower lobes) or if the bronchial mucosa is friable. The procedure can be carried out under sedation or general anaesthesia, depending on resources and physician/institution preference. Some patients report an improvement in their asthma after the first treatment. Although carrying out bronchial thermoplasty may seem straightforward, this treatment requires expertise in asthma management and interventional bronchoscopy. In addition, a multidisciplinary team combining physicians with a specific interest in interventional endoscopy and those with an expertise in chronic airway disorders will ensure the best possible care to the most appropriate patient.

\section{Is it efficacious?}

After an initial study exploring the safety and clinical effects of bronchial thermoplasty in patients with mild to moderate asthma [9], three randomised clinical trials were carried out in patients with moderate to severe asthma [5-7]. The AIR Trial was a randomised, controlled study in 112 asthma patients (56 received bronchial thermoplasty and 56 received standard-of-care) who required treatment with ICS and LABA. The principal outcome measure was the average frequency of mild exacerbations during the LABA withdrawal periods. The Research in Severe Asthma (RISA) Trial was a randomised, controlled safety study in 32 severe patients whose asthma was uncontrolled despite high-dose ICS (15 patients received bronchial thermoplasty and 17 continued with usual care) [5]. After the initial evaluation, there was forced steroid withdrawal to attempt to wean patients from ICS and OCS between weeks 22 and 36. The largest study, AIR2 [7], was a double-blinded, randomised, sham-controlled study that enrolled patients who had uncontrolled asthma

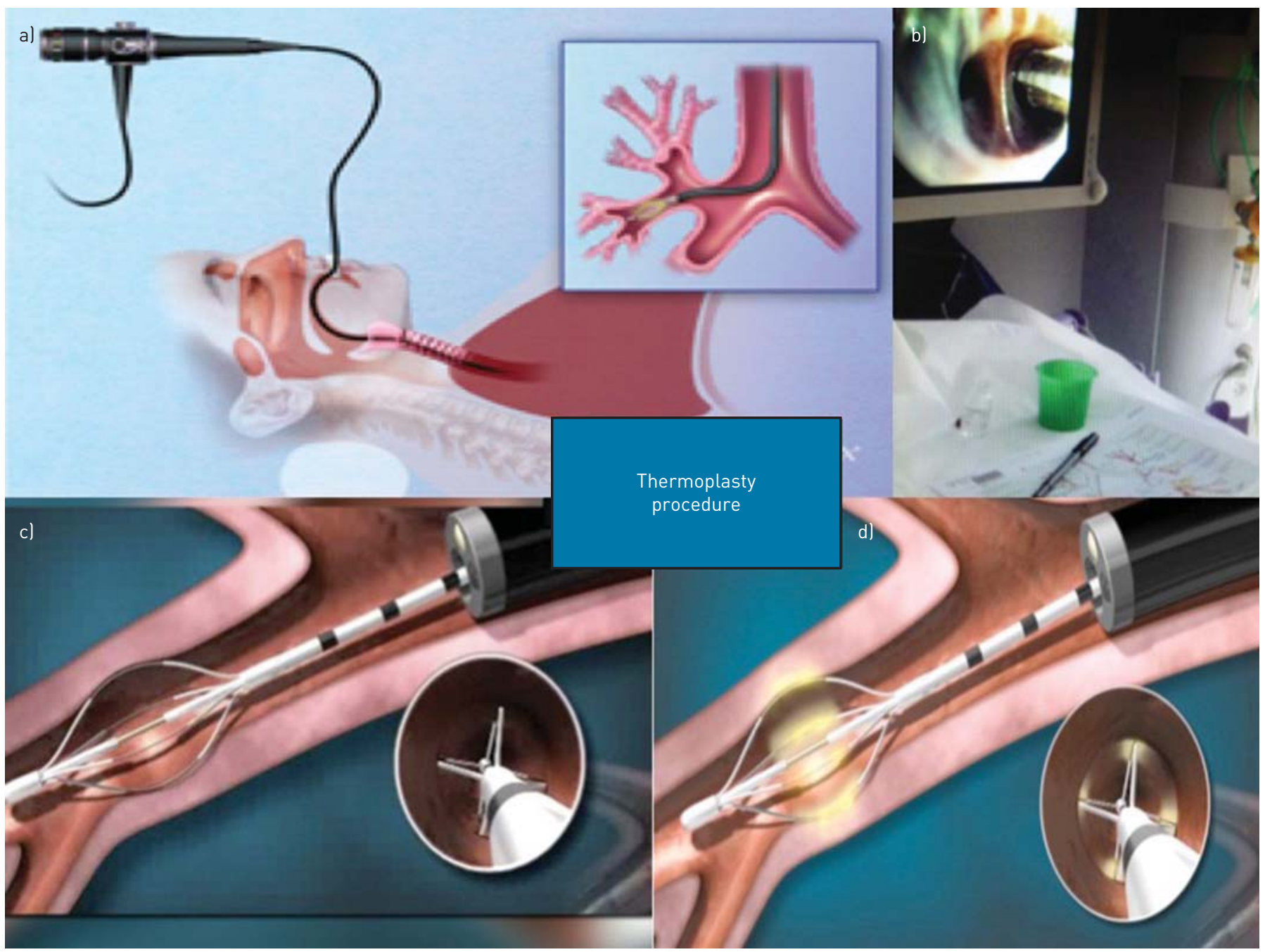

FIGURE 1 Bronchial thermoplasty procedure. a) Bronchial thermoplasty is performed through fibreoptic bronchoscopy. b) A catheter is inserted in a proximal bronchus. c) The catheter is opened in situ. d) The catheter delivers controlled thermal energy. 
TABLE 1 Inclusion criteria in studies into bronchial thermoplasty

Feasibility study [9]

$\geqslant 18$
$\leqslant 1500$ or $200-1800$
$0-100$
0
$60-85$

AIR study [6]

$18-65$
$\geqslant 200$
$\geqslant 100$
0
$60-85$

RISA study [5]

$18-65$
$>1500$
$\geqslant 100$
$\leqslant 30$
$\geqslant 50$

AIR2 study [7]

$$
\begin{gathered}
18-65 \\
>1000 \\
\geqslant 100 \\
\leqslant 10 \\
\geqslant 60
\end{gathered}
$$

AIR: Asthma Intervention Research; RISA: Research in Severe Asthma; ICS: inhaled corticosteroids; LABA: long-acting $\beta_{2}$-agonist; OCS: oral corticosteroids; BD: bronchodilator; FEV1: forced expiratory volume in $1 \mathrm{~s}$. \#: beclomethasone dipropionate equivalent; ${ }^{\circledR}$ : inclusion criteria different at the two participating sites (Narinder Shargill, Boston Scientific, San Jose, CA, USA; personal communication).

despite high-dose ICS and a LABA. 190 patients were treated with bronchial thermoplasty and 98 received sham thermoplasty. The sham thermoplasty treatment reproduced all the audio and visual signals of the technique but the catheter did not deliver any radiofrequency energy. The treatment was administered by an unblinded bronchoscopy team and all the assessments and follow-up visits were conducted by a blinded team. The primary outcome measure was change from baseline in average group mean Asthma Quality of Life Questionnaire (AQLQ) score. Details of these trials are summarised in tables 1 and 2.

\section{Effects on quality of life}

In the AIR study, AQLQ improved significantly in comparison with the control group [6]. In the RISA study, although bronchial thermoplasty-treated subjects were on reduced maintenance therapy following the steroid wean phase, they showed an improvement in their quality of life compared with control subjects [5]. In AIR2, compared with sham control, the bronchial thermoplasty-treated group demonstrated superior AQLQ scores as well as a greater proportion with a clinically meaningful improvement in AQLQ $(\geqslant 0.5)$ in the intent-to-treat and per-protocol groups (79\% versus $64 \%$ and $81 \%$ versus $63 \%$; posterior probability of superiority (PPS) $99.6 \%$ and $99.9 \%$, respectively) [7].

\section{Effects on asthma control}

In the initial feasibility study, Cox et al. [9] reported an improvement in the number of symptom-free days

\begin{tabular}{|c|c|c|c|c|c|c|}
\hline Study & Features & $\begin{array}{l}\text { Duration } \\
\text { weeks }\end{array}$ & Assessments & Inclusion criteria & Subjects $n$ & Control \\
\hline RISA [5] & $\begin{array}{l}\text { Multicentre, } \\
\text { randomised, } \\
\text { parallel groups }\end{array}$ & 52 & $\begin{array}{c}\text { Changes: ICS, OCS, PEFR, FEV1, } \\
\text { AQLQ, safety }\end{array}$ & $\begin{array}{l}\text { Age } 18-65 \text { years, ICS } \\
>750 \mu \mathrm{g}, \mathrm{FP}+\mathrm{LABA} \\
\text { pre-BD } \mathrm{FEV} 1 \geqslant 50 \% \\
\text { pred, uncontrolled }\end{array}$ & 32 & $\begin{array}{l}\text { Continue usual } \\
\text { ICS+LABA }\end{array}$ \\
\hline AIR [6] & $\begin{array}{l}\text { Multicentre, } \\
\text { randomised, } \\
\text { parallel groups }\end{array}$ & 52 & $\begin{array}{l}\text { Changes: ICS, OCS, rescue } \beta_{2} \\
\text { agonists, exacerbations, PEFR, } \\
\text { FEV1, AQLQ, safety during LABA } \\
\text { withdrawal periods }\end{array}$ & $\begin{array}{c}\text { Age } 18-65 \text { years, ICS } \\
\geqslant 200 \mu \mathrm{g}, \mathrm{BDP}+\mathrm{LABA}, \\
\text { pre-BD FEV1 } 60-85 \% \\
\text { pred }\end{array}$ & 109 & $\begin{array}{l}\text { Continue usual } \\
\text { ICS+LABA }\end{array}$ \\
\hline AIR2 [7] & $\begin{array}{l}\text { Multicentre, } \\
\text { randomised, } \\
\text { placebo, } \\
\text { double-blind, } \\
\text { parallel groups }\end{array}$ & 52 & $\begin{array}{c}\text { Number of asthma-free days, } \\
\text { exacerbations ladmissions, } \\
\text { urgent care), rescue } \beta_{2} \text { agonists, } \\
\text { PEFR, FEV1, AQLQ, absenteeism, } \\
\text { safety }\end{array}$ & $\begin{array}{c}\text { Age } 18-65 \text { years, ICS } \\
>1000 \mu \mathrm{g} \\
\text { BDP+LABA, pre-BD } \\
\text { FEV1 } \geqslant 60 \% \text { pred }\end{array}$ & 288 & $\begin{array}{l}\text { Three sham sessions of } \\
\text { bronchial thermoplasty } \\
\text { every } 3 \text { weeks, } \\
\text { continue usual } \\
\text { ICS+LABA }\end{array}$ \\
\hline
\end{tabular}
in the 3 months following bronchial thermoplasty. In AIR, asthma control improved after bronchial thermoplasty; subjects had more symptom-free days and a significant improvement in quality of life [6]. Furthermore, the bronchial thermoplasty-treated subjects used less rescue medication, equating to

\section{TABLE 2 Main characteristics of studies into severe asthma}

RISA: Research in Severe Asthma; AIR: Asthma Intervention Research; ICS: inhaled corticosteroids; OCS: oral corticosteroids; PEFR: peak expiratory flow rate; FEV1: forced expiratory volume in $1 \mathrm{~s} ; A Q L Q$ : Asthma Quality of Life Questionnaire; FP: fluticasone propionate; LABA: longacting $\beta_{2}$-agonist; $\mathrm{BD}$ : bronchodilator; BDP: beclomethasone dipropionate. For all three studies, thermoplasty treatment consisted of three sessions of bronchial thermoplasty every 3 weeks, while continuing usual ICS+LABA. 
approximately two fewer canisters of short-acting bronchodilators per year. In RISA, the bronchial thermoplasty-treated group demonstrated improved asthma control and reduced use of rescue medications prior to the forced steroid withdrawal period [5]. Despite a reduction in medication following the steroid wean phase, the bronchial thermoplasty-treated group continued to show reduced use of short-acting bronchodilators, more symptom-free days and maintenance of an improved Asthma Control Questionnaire (ACQ) score. AIR2 saw a significant reduction in the number of bronchial thermoplasty-treated subjects with worsening asthma compared with sham in the post-treatment period $(27.3 \%$ versus $42.9 \%$, respectively; PPS 99.7\%) [7]. There was no difference in the number of symptom-free days, use of rescue medications or the total asthma symptom score.

Effects on asthma exacerbations, use of urgent care and absences from work or school

In AIR, the bronchial thermoplasty-treated group presented, on average, fewer mild exacerbations during the LABA withdrawal period at 1 year than the control group [6]. In AIR2, compared with sham control, the bronchial thermoplasty-treated group demonstrated superiority, with fewer severe exacerbations (-32\%; PPS 95.5\%), visits to urgent care (-84\%; PPS 99.9\%) and days absent from work or school (-66\%; PPS 99.3\%) [7].

\section{Effects on lung function and airway responsiveness}

The feasibility study reported improvements in expiratory flow and airway responsiveness 3 and 12 months after bronchial thermoplasty [9]. These improvements persisted for up to 3 years, although this finding was not significant due to the high number of patients lost to follow-up, especially in the untreated group. In AIR, morning peak flow measurements were better than in the control group [6]. In AIR2, no change in respiratory function was noted at 1 year post treatment, but there was a reduction in steroid consumption [7]. In fact, the effects of bronchial thermoplasty on asthma were comparable to those of omalizumab, with improvements in exacerbation rate and quality of life but little or no effect on respiratory function [10].

\section{Sparing effect on corticosteroid therapy}

In RISA, the use of OCS and ICS fell by $63.5 \%$ and $28.6 \%$, respectively, in bronchial thermoplasty-treated subjects, compared with $26.2 \%$ and $20 \%$ in the control group. In spite of reduced maintenance therapy, significant improvements were seen in quality of life and asthma symptom scores 4 months after the steroid wean phase [5].

\section{Effects on lung morphology assessed by imaging}

Airway imaging is a minimally invasive modality for assessing the efficacy and safety of bronchial thermoplasty. A study using computed tomography (CT) in canine models has been carried out by BROwN et al. [11]. Some authors have looked at the effects of bronchial thermoplasty on bronchial calibre, bronchial sensitivity to methacholine and response to deep inspiration [12]. Measurements at 5 weeks after treatment suggested reduced airway hyperresponsiveness with no change in lung compliance. In the feasibility study, 16 subjects were assessed by high-resolution CT (HRCT) at baseline, 1 year and 2 years; there was no consistent evidence for bronchiectasis, bronchial wall thickening or other structural abnormalities of the bronchi or parenchyma [9]. The results at 5 years in the 93 AIR2 subjects for whom initial and post-treatment HRCT imaging was available showed either no change or improvement in $82 \%$ of cases. Worsening or newly developed bronchiectasis was reported in only three (3\%) patients [7]. There is no mention of the mosaic pattern that can point to constrictive bronchiolitis, bronchial stenosis or emphysema [2].

Data on the effects of bronchial thermoplasty are still quite limited; two of the randomised clinical trials were not blinded. Furthermore, these results are difficult to generalise to the real-life severe asthma population, as many will have characteristics outside of the clinical study eligibility criteria. Overall, bronchial thermoplasty demonstrated improved quality of life but there was a large sham effect, a common finding in studies exploring quality of life in severe asthma [10]. The beneficial effects of bronchial thermoplasty on quality of life and asthma control as well as a reduction in doses of ICS or OCS in randomised trials are consistent. Bronchial thermoplasty treatment also appears to lead to a decrease in airway hyperresponsiveness without any changes in respiratory function. A recent analysis showed that the beneficial effects of bronchial thermoplasty seem to last for at least 5 years $[2,13,14]$; however, studies over a longer term are needed.

\section{What is the safety profile?}

Adverse events observed during the treatment phase (up to 6 weeks after the final bronchoscopy) and the post-treatment phase (from 6 weeks after the final bronchoscopy) have been described for each of the four clinical trials (table 3): the feasibility study in mild to moderate asthma, AIR in moderate to severe asthma [6], 
AIR2 in moderate to severe asthma [7] and RISA in severe asthma patients. In three of the studies, longer-term adverse events up to 5 years have been described $[2,14,15]$.

\section{Adverse events in the initial treatment period}

Bronchial thermoplasty-treated subjects presented with more symptoms typical of asthma (e.g. cough, wheeze, expectoration, dyspnoea, nocturnal awakening) than control subjects, and occasionally had general symptoms such as fever in the hours following treatment. These symptoms usually resolved after 7 days but led to hospital admission in $3.4 \%$ of bronchoscopies in moderate to severe asthma in AIR2 and $15.6 \%$ of severe asthma cases in RISA (table 3). This finding underlines the importance of optimising asthma control prior to the first bronchial thermoplasty treatment and ensuring close monitoring in the days following treatment. In AIR2, one patient presented significant haemoptysis from the right upper lobe 1 month after the last session and required bronchial artery embolisation. Slight bleeding had been observed during the treatment of this lobe and this complication was considered to be probably related to the treatment [7].

\section{Adverse events at 1 year and in the longer term}

Asthma is associated with structural and inflammatory changes of the bronchial mucosa [16], and the application of controlled thermal energy $\left(65^{\circ} \mathrm{C}\right)$ raises the question of whether bronchial thermoplasty may induce further injury to the bronchial wall. After long-term follow-up in the four clinical trials, there was no evidence for treatment-related airway stenosis or bronchiectasis. None of the trials documented any bronchial damage at 1 year of follow-up. One case of pulmonary abscess developed 14 months after treatment in AIR2 [13]. The affected upper left lobe underwent surgical resection and no bronchial abnormality was documented on histological examination. The abscess was considered to be secondary to a respiratory infection with no clear connection to bronchial thermoplasty.

During this 5-year follow-up, pre-bronchodilator forced expiratory volume in $1 \mathrm{~s}$ (FEV1) remained stable in the two studies of patients with moderate to severe asthma [2, 14]. Additionally, chest radiography [13] and HRCT at 3 and 5 years did not show any significant structural changes other than transient and temporary abnormalities typically seen in moderate to severe asthma [2]. In RISA, follow-up of chest radiographs at 5 years was performed in the small group with the most severe asthma; no significant changes were documented, FEV1 did not deteriorate, and the number of hospital admissions for exacerbation of asthma had fallen over this period [14].

\section{Who is a candidate for bronchial thermoplasty?}

Clinical studies of bronchial thermoplasty in adults with asthma have addressed a broad range of asthma patient severity, from mild to severe. Although it is true that most patients presented with inadequate control of their asthma and most had evidence of airways obstruction, maintenance asthma treatments and ICS doses were variable. Moreover, some patients received OCS as well [5]. Bronchial thermoplasty has been administered in clinical trials in difficult (uncontrolled) cases of asthma but not exclusively in those at the extreme end of the severity spectrum (table 3).

\section{ICS dose}

There may be a tendency to reserve bronchial thermoplasty for patients whose asthma is uncontrolled despite the combination of high-dose ICS and LABA. The required daily dose of ICS prior to considering bronchial thermoplasty is not entirely clear; benefits were seen in uncontrolled subjects despite a daily dose of fluticasone $>750 \mu \mathrm{g}$ in RISA [5], beclomethasone $>1000 \mu \mathrm{g}$ in AIR2 [7], and moderate doses of ICS in other studies [9]. We recommend that medical treatment be optimised before offering bronchial thermoplasty.

\section{Lung function}

In the clinical development programme, FEV1 had been considered when assessing subjects for eligibility. This was justified by the increased incidence of hospitalisation following bronchoscopy for bronchial thermoplasty in subjects whose asthma is very severe (15.6\% in RISA versus $3.4 \%$ in AIR2). The AIR2 study required a pre-bronchodilator FEV1 of $>60 \%$ of predicted versus at least $50 \%$ of predicted in RISA for eligibility.

\section{Airways hyperresponsiveness}

The clinical programme included patients that were selected at least in part due to evidence for bronchial hyperreactivity expressed as either a significant response to $\beta_{2}$ agonists, deterioration on withdrawal of $\beta_{2}$ agonists or increased responsiveness to methacholine. For example, eligibility criteria in AIR included a bronchodilator response of $12 \%$ after $400 \mu \mathrm{g}$ of inhaled salbutamol or a positive methacholine challenge 


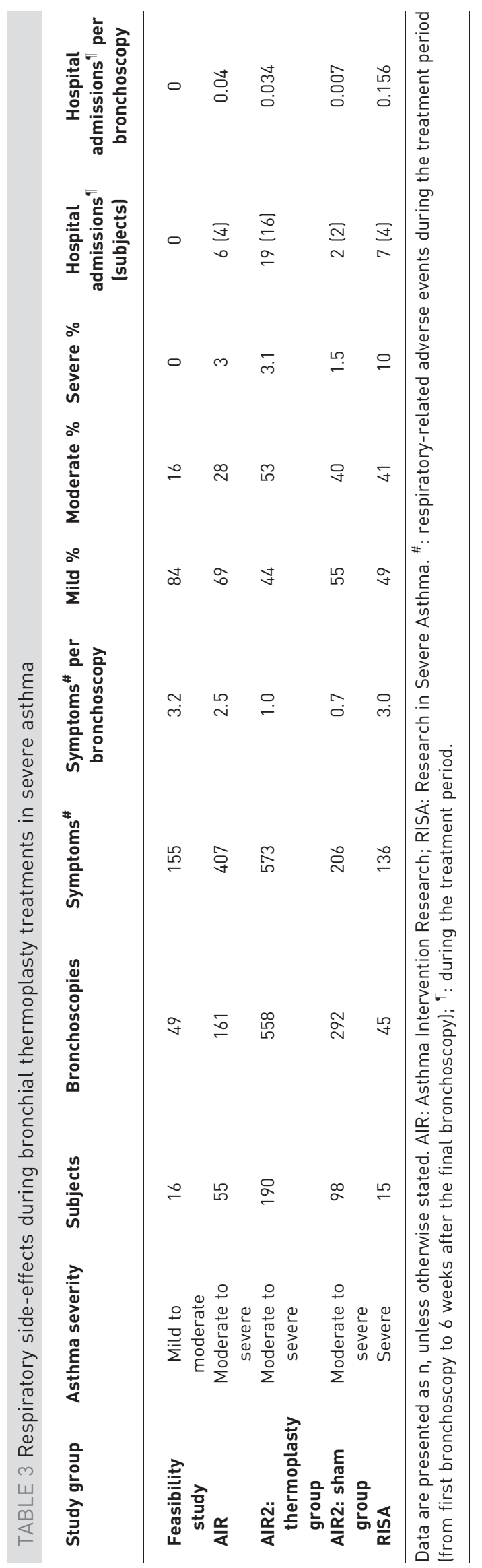


test defined as $<8 \mathrm{mg} \cdot \mathrm{mL}^{-1}$ [6]. In addition, in patients who were already treated with a combination of ICS and LABA, a significant functional or uncontrolled deterioration in the 2 weeks after stopping the LABA was required as well. In the real world, measuring bronchial hyperresponsiveness using a challenge test prior to bronchial thermoplasty may be problematic in severe asthma, especially when baseline respiratory function is compromised.

\section{Absence of asthma control}

Bronchial thermoplasty is indicated in cases of asthma that are inadequately controlled or uncontrolled despite maximal medical therapy; this defines difficult asthma. Asthma control can be assessed in various ways, including by frequency of exacerbations and by composite questionnaires such as the Asthma Control Test and ACQ (based on recent daily symptoms) [17]. The effects of bronchial thermoplasty on reduction in exacerbations were very clearly shown in AIR2 during the post-treatment period [7]; however, it may not be essential to have a history of severe exacerbations to benefit from bronchial thermoplasty. Asthma that remains uncontrolled despite maximal medical therapy is consistent with the patient population studied in the clinical programme.

\section{Contraindications}

Patients with a history of life-threatening asthma were excluded from the clinical programme. Similarly, both three or more hospital admissions or four or more courses of systemic corticosteroids in the year preceding bronchial thermoplasty were exclusion criteria in AIR2 [13]. Heavy use of short-acting bronchodilators $\left(>4\right.$ puffs $\cdot$ day $\left.^{-1}\right)$ was also an exclusion criterion for AIR2. The procedure poses a theoretical risk of infection. It seems reasonable to exclude patients with a history of three or more proven respiratory infections treated with antibiotics in the previous year [6]. Because of the theoretical risk of infection, bronchiectasis is at present an absolute contraindication to bronchial thermoplasty. Chronic rhinosinusitis was a contraindication for the AIR and AIR2 studies, presumably because of the difficulties distinguishing clinical events associated with rhinosinusitis from asthma [7]. Subjects with a smoking history of $>10$ pack-years were excluded from the clinical programme; no information on the safety or effectiveness of bronchial thermoplasty in this patient population is available.

\section{Responsive phenotypes}

We might imagine that the patients who benefit the most from bronchial thermoplasty would be those who have greater airway hyperreactivity as demonstrated by response to $\beta_{2}$ agonists, response to methacholine challenge or tendency to frequent exacerbations. In the same way, we might think that bronchial thermoplasty would be even more effective in those where smooth muscle contraction plays an important role in their disease; however, there are no clinical data to support this assumption. Similarly, there is little evidence that the inflammatory phenotype is relevant to clinical response. Even if we believe that airway hyperresponsiveness is an important criterion for response to bronchial thermoplasty, there is only a weak relationship between eosinophilic inflammation and airway hyperresponsiveness to direct stimuli [18]. Likewise, the presence or absence of allergies by self-report or other predisposing factors does not seem to be a factor that can predict response to bronchial thermoplasty [2].

\section{How does it work?}

Bronchial thermoplasty is a procedure that delivers controlled thermal energy at $65^{\circ} \mathrm{C}$ to airways between 3 and $10 \mathrm{~mm}$ diameter via a bronchoscope under direct visual guidance (fig. 1). There are a number of possible mechanisms of action that alone, or in combination, might explain the beneficial effects of bronchial thermoplasty. To date, no unique mechanism has been formally identified.

Because an increase in airway smooth muscle (ASM) mass is one of the major characteristics of airway remodelling in severe asthma patients [16], the first and most well documented mechanism of action concerns reduction in the bronchial wall smooth muscle [19]. A number of reports have emphasised the effects of increased smooth muscle mass in severe asthma, particularly on airway hyperresponsiveness and permanent airflow limitation [20, 21]. A reduction in ASM mass after bronchial thermoplasty has been observed experimentally in a canine model [11] and in nonasthmatic human lobectomy specimens examined between 1 and 3 weeks after bronchial thermoplasty [22]. This reduction in ASM was directly linked in dogs to a decrease in airway hyperresponsiveness; however, reduction in airway hyperresponsiveness in asthmatic human subjects after bronchial thermoplasty was inconsistent [13].

A second mechanism might involve a direct effect on the contractile properties of the ASM or a stiffening of the bronchial wall to which the smooth muscle attaches. The effects of the application of various temperatures on in vitro contraction of bovine bronchial smooth muscle have been examined. Acetylcholine-induced contraction of bronchial smooth muscle was inhibited at temperatures exceeding $55^{\circ} \mathrm{C}$, 
while relaxation mediated by a $\beta_{2}$ agonist was not affected, suggesting that bronchial thermoplasty may alter the contractile properties of ASM cells [23]. Interactions between actin and myosin were also very sensitive to temperature, and changes occurred in the absence of any histological signs of necrosis or apoptosis. It is unclear whether the acute phenomena observed in vitro may persist in the long term in vivo and whether they may be the cause of reduced airway contractility.

Other potential mechanisms of action include decreased secretion of inflammatory mediators by ASM cells and changes to the epithelium of the airways, the nerve endings or the function of the inflammatory cells that infiltrate the bronchial mucosa.

Currently, the mechanisms of action for bronchial thermoplasty are poorly understood. Aside from the issue of reduced ASM mass, many questions remain (see online supplementary material for a list). None of the potential mechanisms mentioned have been studied either in preclinical experimental models of asthma or in clinical studies, and more work is needed in order to elucidate the various potential mechanisms of action of bronchial thermoplasty. Ultimately, this understanding may allow for improved appropriate patient selection.

\section{Acknowledgements}

The authors thank Caroline Benet and Nejma Saidani (Boston Scientific Corporation, Voisins-le-Bretonneux, France), and Neil Inhaber and Robin Goodrich (Boston Scientific Corporation, Marlborough, MA, USA) for their editorial assistance and helpful discussion and suggestions.

\section{References}

1 Chung KF, Wenzel SE, Brozek JL, et al. International ERS/ATS guidelines on definition, evaluation and treatment of severe asthma. Eur Respir J 2014; 43: 343-373.

2 Wechsler ME, Laviolette M, Rubin AS, et al. Bronchial thermoplasty: long-term safety and effectiveness in patients with severe persistent asthma. J Allergy Clin Immunol 2013; 132: 1295-1302.

3 Boulet LP, Laviolette M. Is there a role for bronchial thermoplasty in the treatment of asthma? Can Respir J 2012; 19: 191-192.

Mayse ML, Laviolette M, Rubin AS. Clinical pearls for bronchial thermoplasty. J Bronchol 2007; 14: 115-123.

Pavord ID, Cox G, Thomson NC, et al. Safety and efficacy of bronchial thermoplasty in symptomatic, severe asthma. Am J Respir Crit Care Med 2007; 176: 1185-1191.

6 Cox G, Thomson NC, Rubin AS, et al. Asthma control during the year after bronchial thermoplasty. N Engl J Med 2007; 356: 1327-1337.

7 Castro M, Rubin AS, Laviolette M, et al. Effectiveness and safety of bronchial thermoplasty in the treatment of severe asthma: a multicenter, randomized, double-blind, sham-controlled clinical trial. Am J Respir Crit Care Med 2010; 181: 116-124.

8 Boulet LP, FitzGerald JM, Levy ML, et al. A guide to the translation of the Global Initiative for Asthma (GINA) strategy into improved care. Eur Respir J 2012; 39: 1220-1229.

9 Cox G, Miller JD, McWilliams A, et al. Bronchial thermoplasty for asthma. Am J Respir Crit Care Med 2006; 173: 965-969.

10 Humbert M, Beasley R, Ayres J, et al. Benefits of omalizumab as add-on therapy in patients with severe persistent asthma who are inadequately controlled despite best available therapy (GINA 2002 step 4 treatment): INNOVATE. Allergy 2005; 60: 309-316.

11 Brown RH, Wizeman W, Danek C, et al. In vivo evaluation of the effectiveness of bronchial thermoplasty with computed tomography. J Appl Physiol 2005; 98: 1603-1606.

12 Brown RH, Wizeman W, Danek C, et al. Effect of bronchial thermoplasty on airway distensibility. Eur Respir J 2005; 26: $277-282$.

13 Castro M, Rubin A, Laviolette M, et al. Persistence of effectiveness of bronchial thermoplasty in patients with severe asthma. Ann Allergy Asthma Immunol 2011; 107: 65-70.

14 Pavord ID, Thomson NC, Niven RM, et al. Safety of bronchial thermoplasty in patients with severe refractory asthma. Ann Allergy Asthma Immunol 2013; 111: 402-407.

15 Thomson NC, Rubin AS, Niven RM, et al. Long-term (5 year) safety of bronchial thermoplasty: Asthma Intervention Research (AIR) trial. BMC Pulm Med 2011; 11: 8.

16 Jeffery PK. Remodeling in asthma and chronic obstructive lung disease. Am J Respir Crit Care Med 2001; 164: S28-S38.

17 Reddel HK, Taylor DR, Bateman ED, et al. An official American Thoracic Society/European Respiratory Society statement: asthma control and exacerbations: standardizing endpoints for clinical asthma trials and clinical practice. Am J Respir Crit Care Med 2009; 180: 59-99.

18 Louis R, Sele J, Henket M, et al. Sputum eosinophil count in a large population of patients with mild to moderate steroid-naive asthma: distribution and relationship with methacholine bronchial hyperresponsiveness. Allergy 2002; 57: 907-912.

19 Cox PG, Miller J, Mitzner W, et al. Radiofrequency ablation of airway smooth muscle for sustained treatment of asthma: preliminary investigations. Eur Respir J 2004; 24: 659-663.

20 Benayoun L, Druilhe A, Dombret MC, et al. Airway structural alterations selectively associated with severe asthma. Am J Respir Crit Care Med 2003; 167: 1360-1368.

21 Solway J, Irvin CG. Airway smooth muscle as a target for asthma therapy. N Engl J Med 2007; 356: 1367-1369.

22 Miller JD, Cox G, Vincic L, et al. A prospective feasibility study of bronchial thermoplasty in the human airway. Chest 2005; 127: 1999-2006.

23 Dyrda P, Tazzeo T, DoHarris L, et al. Acute response of airway muscle to extreme temperature includes disruption of actin-myosin interaction. Am J Respir Cell Mol Biol 2011; 44: 213-221. 